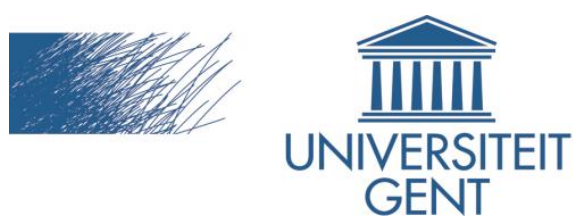

biblio.ugent.be

The UGent Institutional Repository is the electronic archiving and dissemination platform for all UGent research publications. Ghent University has implemented a mandate stipulating that all academic publications of UGent researchers should be deposited and archived in this repository. Except for items where current copyright restrictions apply, these papers are available in Open Access.

This item is the archived peer-reviewed author-version of:

NinSuna: Metadata-driven Media Delivery

Davy Van Deursen, Wim Van Lancker, Erik Mannens, and Rik Van de Walle

In Proceedings of the 3th European Conference, ServiceWave 2010, pp 225-226, December, 2010

To refer to or to cite this work, please use the citation to the published version:

D. Van Deursen, W. Van Lancker, E. Mannens, and R. Van de Walle (2010). NinSuna: Metadata-driven Media Delivery. In Proceedings of the 3th European Conference, ServiceWave 2010, pp. 225-226, December 2010, Ghent, Belgium. 


\title{
NinSuna: Metadata-driven Media Delivery
}

\author{
Davy Van Deursen*, Wim Van Lancker, Erik Mannens, and Rik Van de Walle \\ Ghent University - IBBT, ELIS - Multimedia Lab, Belgium \\ davy.vandeursen@ugent.be
}

\section{Platform Overview}

Today, delivery of multimedia content introduces a number of important challenges due to the growing amount of multimedia content on the one hand and the growing diversity in usage environments on the other hand. Furthermore, we need to deal with a growing amount of media formats used for compressing and packaging multimedia content.

In order to deal with such a heterogeneous multimedia landscape, we developed NinSuna [3], which is a fully integrated metadata-driven media delivery platform. Its basic design is inspired by the principles of XML-driven content adaptation techniques, while its final design and the implementation thereof are based on Semantic Web technologies such as the Resource Description Framework (RDF), Web Ontology Language (OWL), and SPARQL Protocol And RDF Query Language (SPARQL). Furthermore, a tight coupling exists between the design of the media delivery platform and a model for describing structural, content, and scalability information of media bitstreams, enabling a formatindependent adaptation and packaging approach [1].

\section{Features}

Our media delivery platform is characterized by a number of features. First of all, the core software modules of the platform (i.e., selection, adaptation, and packaging of media bitstreams) are fully independent of media formats (i.e., both coding and delivery formats). Therefore, the platform is highly extensible, since new media formats can be added by means of plugins. Also, the different platform modules can be distributed over different machines, which makes the platform scalable.

NinSuna uses a central multimedia ontology which couples the served media resources with any available metadata. Hence, the metadata is stored into a fully RDF-based storage backend for descriptive metadata which is accessible through a SPARQL endpoint. Also, a number of metadata parsers are provided for importing and converting XML-based metadata to an enhanced

\footnotetext{
* The research activities as described in this paper were funded by Ghent University, the Interdisciplinary Institute for Broadband Technology (IBBT), the Institute for the Promotion of Innovation by Science and Technology in Flanders (IWT), the Fund for Scientific Research Flanders (FWO-Flanders), and the European Union.
} 
RDF representation (e.g., NewsML) while parsers for other metadata schemes can be added as plugins.

The platform supports various forms of content adaptation: scenes or shots can be extracted from any media resource to facilitate fine-grained search queries, requested media fragments can be adapted depending on the scalability provisions in the media stream, and track combinations can be selected (e.g., audio/video stream selection based on bit rate). Further, the platform supports the most common media delivery formats on the Web: streaming media delivery through RTSP and RTMP, HTTP progressive download (MP4, Ogg, 3GPP, MPEG-2 TS), and HTTP Live streaming.

Finally, NinSuna is a server-side reference implementation of the W3C Media Fragments URI 1.0 specification [2], which mission is to address media fragments on the Web using Uniform Resource Identifiers (URIs). Having global identifiers for arbitrary media fragments allows substantial benefits, including linking, bookmarking, caching, and indexing.

\section{Demonstration}

We built two front-end applications to demonstrate our media delivery platform. The first demonstration consists of a faceted browser facilitating the retrieval of news fragments ${ }^{1}$. More specifically, media resources representing news broadcasts are annotated on a scene level, by using NewsML metadata serialized in RDF. Hence, we can use the faceted browsing paradigm to let the end-user obtain his/her desired news fragments. Once a news fragment is chosen, the selected media fragment is extracted, packaged, and sent to the client. Additionally, dynamic frame rate adaptations are demonstrated as well.

The second demonstration shows the interaction between NinSuna and Media Fragment 1.0 URIs ${ }^{2}$. More specifically, we show which HTTP requests/responses are sent to/from our platform and how the user agents can visualize such media fragment URIs.

\section{References}

1. D. Van Deursen et al.: Format-independent and Metadata-driven Media Resource Adaptation using Semantic Web Technologies. Multimedia Systems 16(2), 85-104 (March 2010)

2. D. Van Deursen et al.: Implementing the Media Fragments URI Specification. In: $19^{\text {th }}$ International Conference on World Wide Web, WWW 2010. pp. 1361-1364. Raleigh, North Carolina, USA (April 2010)

3. D. Van Deursen et al.: NinSuna: a Fully Integrated Platform for Format-independent Multimedia Content Adaptation and Delivery based on Semantic Web Technologies. Multimedia Tools and Applications - Special Issue on Data Semantics for Multimedia Systems 46(2-3), 371-398 (January 2010)

\footnotetext{
${ }^{1}$ Screen cast available at http://ninsuna.elis.ugent. be/NinSunaFacets\#demo.

${ }^{2}$ Screen cast available at http://ninsuna.elis.ugent.be/MediaFragmentsServer\# screencast.
} 